\title{
LAS ESCUELAS DEL EMPRENDIMIENTO SOCIAL: UNA TRIPLE PROPUESTA TEÓRICA PARA ENFOCAR SU ESTUDIO
}

\author{
Autores: Braulio Pareja Cano ${ }^{1}$ \\ Investigador en formación de la Cátedra de Ética Económica y Empresarial \\ Facultad de Ciencias Económicas y Empresariales \\ Universidad Pontificia Comillas (ICAI-ICADE) \\ Anna Bajo Sanjuán ${ }^{2}$ \\ Profesora e investigadora de la Cátedra de Ética Económica y Empresarial \\ Facultad de Ciencias Económicas y Empresariales \\ Universidad Pontificia Comillas (ICAI-ICADE) \\ José Luis Fernández Fernández ${ }^{3}$ \\ Director de la Cátedra de Ética Económica y Empresarial \\ Profesor ordinario de la Facultad de Ciencias Económicas y Empresariales \\ Universidad Pontificia Comillas (ICAI-ICADE)
}

\footnotetext{
${ }^{1}$ bpareja@icade.comillas.edu

2anna.bajo@icade.comillas.edu

3 jlfernandez@icade.comillas.edu
} 


\title{
Resumen
}

En el ámbito académico aún no existe un consenso para delimitar el concepto de emprendimiento social, y existen diversos enfoques e interpretaciones. Es necesaria una mayor claridad en su definición para diferenciarlo del emprendimiento tradicional. El objetivo principal de este artículo ha sido desarrollar una taxonomía del concepto de emprendimiento social a través de una revisión de la literatura, describiendo tres escuelas de pensamiento, a saber: (i) la escuela de la empresa social, (ii) la escuela del emprendedor social y (iii) la escuela de la innovación social.

Palabras clave: emprendimiento social, empresa social, emprendedor social, innovación social, escuelas de pensamiento.

\begin{abstract}
In academia there is still no consensus in defining the concept of social entrepreneurship, and there are various approaches and interpretations. Clarity in its definition to differentiate it from traditional entrepreneurship is necessary. The main objective of this paper was to develop a taxonomy of the concept of social entrepreneurship through a review of literature, describing three schools of thought, namely: (i) the school of social enterprise, (ii) the school of social entrepreneur and (iii) the school of social innovation.
\end{abstract}

Key words: social entrepreneurship, social enterprise, social entrepreneur, social innovation, schools of thought.

\section{INTRODUCCIÓN}

El interés por el conocimiento en el ámbito del social entrepreneurship, o emprendimiento social en su traducción al castellano, ha crecido enormemente a tenor de los últimos estudios bibliométricos, sobre todo desde los primeros años del siglo XXI (Short y otros, 2009; Peredo y McLean, 2005).

Las lagunas de conocimiento que los investigadores deben abordar son amplias y la mayoría de los autores corroboran la dificultad que existe a la hora de consensuar académicamente una definición de emprendimiento social. Así las cosas, dependiendo de la escuela o el enfoque del grupo de investigación, 
el emprendimiento social se entiende de una u otra manera (Pareja, 2013; Dacin y otros, 2010). Algunos lo justifican por la necesidad de aportar alternativas para generar empleo a la vez que reducir los problemas sociales (Zamagni, 2012). Otros lo enmarcan más en el ámbito institucional, persiguiendo conocer las claves de un management más eficiente en las organizaciones del tercer sector (Shane y Venkataraman, 2000). Los más críticos prevén el advenimiento de un nuevo orden socioeconómico donde la humanidad use del emprendimiento social para enfrentarse a los problemas sociales más graves de nuestro tiempo, como la pobreza, la desigualdad, la defensa del medio ambiente o los derechos humanos (Felberg, 2012; Merino, 2014; Yunus, 2011).

Entre esta heterogeneidad en la aproximación al fenómeno, es necesario una aclaración epistemológica para poder afrontar el estudio del emprendimiento social a partir de una dimensión concreta del conocimiento. La propuesta que se presenta aquí pretende organizar los conceptos a partir de una triple dimensión, que facilite la clasificación de los estudios futuros.

Por eso el enfoque de este estudio es descriptivo. En cuanto a métodos, nos hemos apoyado en la revisión de la literatura para seleccionar los artículos más relevantes. De todos ellos hemos seleccionado los que aportaban una definición más o menos concisa de lo que entendían por emprendimiento social. Estas definiciones las hemos clasificado en tres escuelas diferentes, a saber: la escuela del emprendedor social, la escuela de la empresa social y la escuela de la innovación social.

\section{ESCUELAS DEL EMPRENDIMIENTO SOCIAL}

El fenómeno del emprendimiento social nace a finales de la década de los setenta del siglo XX. Fue Bill Drayton quién acuñó el término "emprendedor social" y fundó la primera organización a nivel mundial dirigida a apoyar a este tipo de emprendedores, la Fundación Ashoka. Su principal objetivo fue y sigue siendo identificar y dar soporte a aquellos que lleven a cabo iniciativas de cambio social (Praszkier y Nowak, 2012). Durante los años ochenta y principios de los noventa, apenas hay menciones académicas concretas al emprendimiento social. A partir de la publicación de Dees (1998), la tendencia cambia, y el horizonte de investigaciones en el ámbito del emprendimiento social aumenta.

El problema principal que se encuentra quien se enfrenta por primera vez con el emprendimiento social es la falta de una definición clara y una descripción ontológica precisa. Se trata de un fenómeno complejo, pues tiene conexión con diversas disciplinas científicas y por tanto es difícil afrontar su investigación de forma holística. Por esta razón creemos que es necesario agrupar las diferentes definiciones en tres escuelas que compartan una misma dimensión taxonómica. 
En un primer lugar encontramos una escuela de pensamiento que habla del emprendimiento social desde el punto de vista del sujeto que emprende, el individuo, es decir el emprendedor social (lo que llamaremos enfoque micro). Suele fijarse en aspectos psicológicos, en rasgos de personalidad y en las motivaciones profundas que explican la iniciativa emprendedora. Otro grupo de investigadores hacen referencia a la organización y todo lo que tiene que ver con ella (enfoque meso): estructura, estrategia, objetivos, modelo de negocio y financiación. Y por último, una tercera escuela se identifica por el tratamiento del proceso de emprendimiento social (enfoque macro), es decir la relación entre la organización, el individuo y el sistema o los sistemas políticos, económicos, y sociales. A partir de esta triple aproximación al concepto, surgen tres abordajes teóricos que cabría considerar como tres diversas y complementarias escuelas (ver esquema 1).

La novedad de nuestra aportación radica sobre todo en introducir el aspecto de innovación social (proceso macro), buscando un abordaje dialéctico que dé una más cumplida inteligencia del fenómeno del emprendimiento social. Junto a los aspectos más comúnmente vistos y analizados por la literatura - los niveles micro y meso-, consideramos que introducir lo referido a la innovación de los procesos sociales contribuye a clarificar el fenómeno en su conjunto. Naturalmente no ha de entenderse que estos tres puntos de referencia sean realidades objetivas independientes y desconectadas entre sí. Por el contrario son polos heurísticos dinámicamente trabados con múltiples puntos de contacto y significada porosidad en sus límites. Sin embargo consideramos que merece la pena caracterizarlos de manera independiente porque ello nos permitirá una comprensión más rica del fenómeno.

El mapa mental que se presenta deriva de la revisión llevada a efecto de la literatura de referencia en el área. Aquellos artículos de corte más humanista también ofrecen justificaciones para acoger esta división. El ser humano tiene una doble dimensión, interna y externa, física y metafísica, pero la empresa también adquiere esas propiedades duales (Martínez, 2005). Según el teorema de la dualidad de estructura de Giddens (1998), la constitución de agentes y la de estructuras no son dos conjuntos de fenómenos dados independientemente, no forman un dualismo sino que representan una dualidad. Esta dualidad necesita de una representación sistémica, que explica entre otras cosas la relación entre ambas y la vinculación con el propio sistema. De aquí nace esta tercera escuela llamada de la innovación social o enfoque macro (figura1). 
Figura 1. Escuelas del emprendimiento social

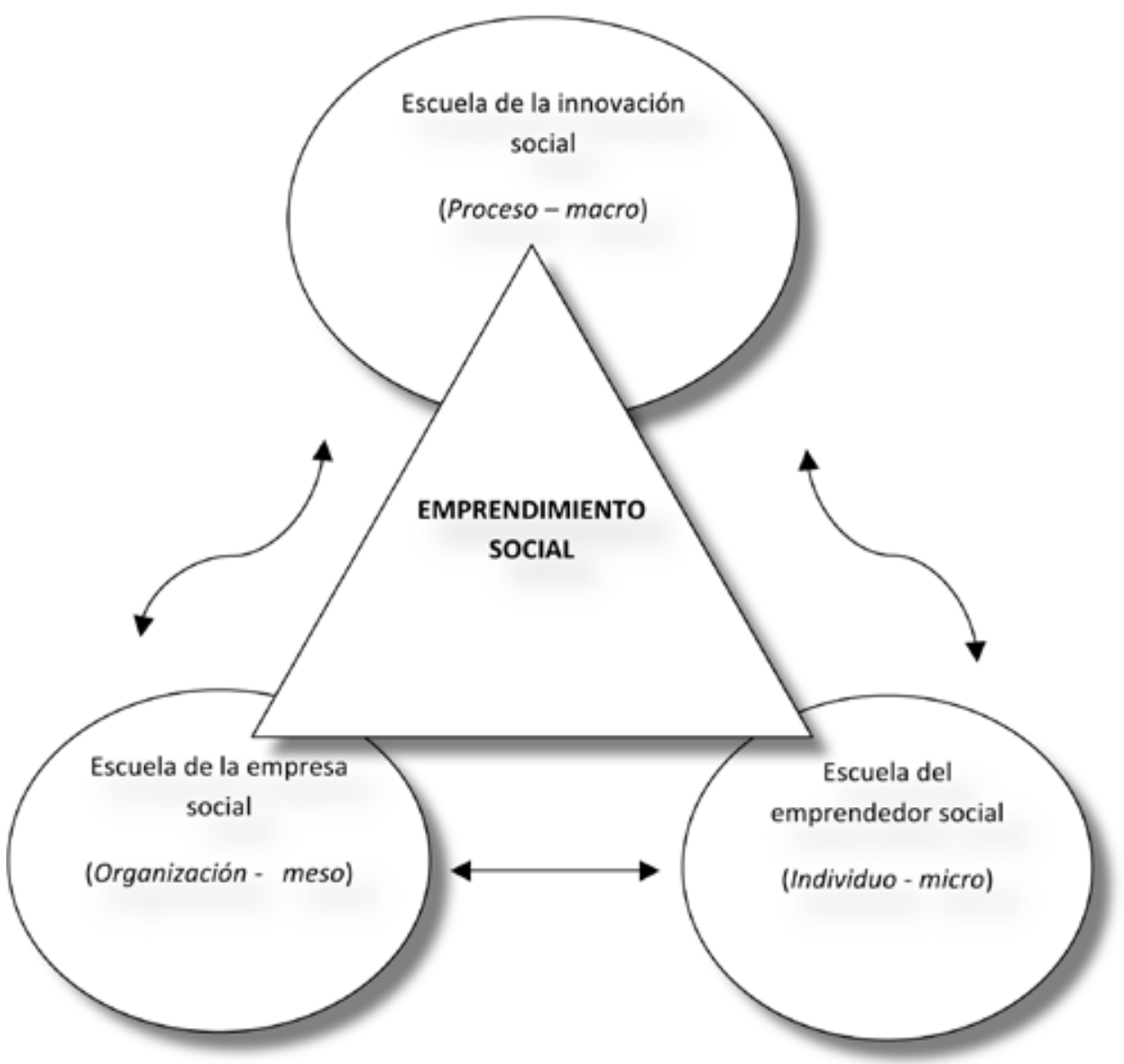

Fuente: elaboración propia a partir del análisis de la literatura.

\subsection{Escuela del emprendedor social}

Esta escuela de pensamiento en el fondo lo que hace es comparar mediante contraste la larga tradición de estudios respecto a la empresa mercantil y el emprendedor tradicional con el fenómeno del emprendimiento social. Este tipo de estudios podría ser el mejor punto de partida para enfocar investigaciones sobre el emprendedor social, adaptando las teorías tradicionales de empresa y gestión a las condiciones y características propias del emprendimiento social.

El economista Schumpeter (1996), fue quien a principios del siglo XX definió al emprendedor por primera vez como un agente económico cuyo leitmotiv era in- 
novar para identificar nuevos mercados e incluso usar de la creatividad y generar nuevas necesidades y modelos de negocio (Pareja, 2013a). A partir de entonces, al emprendedor se le ha considerado un agente económico de primer orden, y ha sido objeto de diversas investigaciones. La mayoría de ellas se centraron en estudiar el comportamiento emprendedor en relación con las habilidades, conductas y competencias específicas (Madden y otros, 1992; Espíritu y Sastre, 2007; Shane y Venkataraman, 2000; Shapero, 1992; Zhao y otros, 2005; Castro y Toro, 1996).

Aunque ya se contaba con algunas referencias anteriores (Waddock y Post, 1991; Campbell, 1998) no es sino hasta finales de la década de los noventa del siglo XX cuando un artículo publicado por Dees (1998) despierta de manera creciente el interés académico por este tema. Por primera vez se plantea la notoriedad de una nueva identidad emprendedora y las diferencias fundamentales entre el emprendedor de negocios (empresario tradicional) y en el ahora denominado emprendedor social como nuevo agente económico.

Según afirma Dees, a la hora de distinguir entre el emprendedor social y el empresario tradicional tanto el uno como el otro son propiamente emprendedores: dos especies dentro del mismo género. Los emprendedores sociales tienen clara conciencia de una misión orientada más que al lucro al impacto social. Es precisamente por ello por lo que se ven abocados a enfrentarse a retos específicos. Para los emprendedores sociales, la misión social es explícita y nuclear en el proyecto y esta circunstancia afecta al modo como identifican las oportunidades de negocio: "Missionrelated impact becomes the central criterion, not wealth creation" (Dees, 1998:2).

Para medir el éxito de un emprendedor tradicional sujeto a la disciplina del mercado habrá que estar atento a la creación de valor económico. Desde este criterio, si el emprendedor considera que hay modo de aplicar los recursos económicos de manera más productiva no dudará en cambiar de negocio. Por lo demás, este tipo de información es fácil reconocer: El mercado competitivo envía mensajes claros al emprendedor tradicional a través del volumen de ventas respecto al modo en que se están consiguiendo los objetivos económicos. Por consiguiente, si se observa que los clientes no compran los productos ofertados, el negocio deja de tener sentido y procede un cambio de estrategia. Sin embargo los mercados no funcionan tan bien con respecto a los emprendedores sociales. Dees señala a este respecto el hecho de que los mercados no son capaces de valorar adecuadamente los resultados del emprendimiento social, cristalizados en ciertas mejoras sociales y en los beneficios obtenidos por parte de quienes no tienen demanda solvente para cubrir sus necesidades. Este indicador de la eficiencia y la eficacia es mucho más débil en este segundo caso, pues es muy difícil medir el valor social creado. Y siendo ello así, los emprendedores sociales tienen dificultad a la hora de conocer el valor económico creado en relación a los recursos utilizados. Ello los aleja un tanto de la disciplina del mercado y hace que se vean con frecuencia abocados a depender de subsidios, donaciones y trabajo voluntario. 
Teniendo lo anterior en cuenta Dees ofrece la siguiente caracterización del emprendedor social a diferencia del emprendedor tradicional. El emprendedor social:

- Adopta la misión de crear y sostener valor social (no sólo valor privado).

- Reconoce y persigue nuevas oportunidades para servir a esta misión.

- Sigue un proceso de innovación, adaptación y aprendizaje continuo.

- Actúa con audacia y sin estar limitado por los recursos que actualmente posee.

- Posee un alto sentido de la responsabilidad hacia los grupos atendidos y los resultados obtenidos (Dees, 1998:4).

El emprendedor social es el agente fundamental y la principal diferencia con el emprendedor tradicional es que el primero desempeña un papel relevante como agente de cambio. Además de valor privado, el emprendedor social genera valor social y posee un sentido alto de la responsabilidad hacia grupos no atendidos o mercados no cubiertos (Dees, 1998). En otros casos, el principal objetivo de un emprendedor social no es generar valor privado, sino generar un impacto social en una comunidad o un grupo social excluido (Neck y otros, 2009).

Para Bornstein (2004), el emprendedor social es aquel que busca soluciones innovadoras a problemas tradicionales y tiene un fuerte compromiso social con la comunidad en la que opera. Esta relación es fundamental para poder comprender no sólo las necesidades a cubrir, sino la propia idiosincrasia de las personas que van a ser atendidas. Para este mismo autor, el emprendedor social no buscaría solamente resolver un problema concreto sino más bien solucionar problemas a gran escala. En ocasiones se sirve para ello de las nuevas tecnologías por el gran impacto transformador que tienen (Sullivan y otros, 2003).

En una línea parecida, Martin y Osberg (2007), relacionan al emprendedor social con alguien cuya principal motivación no es el ánimo de lucro, sino que busca dirigir sus iniciativas hacia sectores sociales poco atendidos y grupos sociales excluidos (Hibbert y otros, 2002; Thompson y otros, 2000). Estos emprendedores buscan causar impacto transformador en la sociedad para resolver problemas desde la raíz. De nuevo tienen la necesidad de generar un cambio sostenible en el tiempo, y no suelen conformarse con una mera actividad asistencial (Zahra y otros, 2009). Un ámbito especialmente proclive a las iniciativas de emprendimiento social es el que apunta hacia algunos de los grandes retos con que nos encontramos en la actualidad, sobre todo el que hace referencia a lo que se viene dando en llamar el problema de la sostenibilidad (Urteaga y Eizagirre, 2011; Benavides y otros, 2012; Cavagnaro y Curiel, 2012; Bajo, 2012).

El emprendedor social persigue encontrar soluciones sostenibles para garantizar un mundo sostenible (Light, 2006). Esto significa buscar fórmulas para ge- 
nerar empresas u otro tipo de organizaciones a través de las cuales sea posible materializar los objetivos propuestos, normalmente alineados con la creación de valor social.

La escuela del emprendedor social que acabamos de delinear en sus aspectos fundamentales no se queda solamente en el estudio y análisis del individuo que emprende. Esta perspectiva se conecta con los planteamientos de las escuelas que describimos a continuación que insisten, por ejemplo, en la capacidad del emprendedor para replicar y extrapolar un proyecto exitoso con fuerte impacto social más allá del ámbito para el que fue inicialmente concebido (Dees y Anderson, 2004; Bloom y Chatterji, 2009; Smith y Stevens, 2010; Deforuny y Nyssens, 2010).

\subsection{Escuela de la empresa social}

La definición de empresa social como organización ha sido abordada por distintos agentes: organismos públicos, gobiernos nacionales, la Comisión Europea y también algunas agencias calificadoras independientes. Cabe destacar la Iniciativa de Emprendimiento Social de la Comisión Europea, donde se define a la empresa social como: "Aquella empresa cuyo objetivo principal es generar un impacto significativo sobre la sociedad, el medio ambiente y la comunidad local" (Comisión Europea, 2011: 5). La propia Comisión incluye a las empresas sociales dentro del marco de la Economía Social, con una larga tradición, sobre todo en cuanto al cooperativismo se refiere (Chávez y Monzón, 2001; Chávez, 2012; Pérez de Mendiguren y otros, 2008).

La empresa social es aquella que con un modelo de negocio sostenible tiene como misión principal generar un impacto y cambio social positivo, usando para ello modelos empresariales innovadores y escalables (Murray y otros, 2011).

Dees y Anderson (2004) afirman que las empresas sociales vendrían a representar una estructura híbrida entre una organización sin ánimo de lucro orientada hacia un objetivo social y una empresa económica en la medida en que utiliza un modelo de negocio que busca la pervivencia sostenible de su actividad a largo plazo. Campbell (1998) por su parte, señala que este tipo de organización tendría la ventaja de poder contar con más capacidad de gestión, con mayores recursos propios y por tanto con mejores posibilidades de éxito y de eficacia en sus acciones.

Las ONGs y el tercer sector en general suelen encontrar dificultades para hacer sostenible su objetivo social debido a su incapacidad para generar recursos a partir de los cuales financiar sus proyectos (Peredo y McLean, 2005; Reis y otros, 2001). Cualquier empresa que opera en el libre mercado hace una serie de estimaciones de beneficios a partir de los cuales toma decisiones determinadas. Las previsiones de las organizaciones sin ánimo de lucro dependen de factores no fáciles de predecir, porque a su vez dependen de otras circunstancias como la 
aprobación de una partida para una subvención gubernamental o el ánimo de caridad de cierto grupo ciudadano (Austin y otros, 2006). La tradición en el ámbito del cooperativismo, ha influido enormemente en la propagación de empresas con una serie de principios comunes como la igualdad y la solidaridad. Esta identidad y principios empresariales tienen reflejo en las diferentes entidades jurídicas de sociedades y otras organizaciones como las asociaciones, mutualidades, cooperativas, cofradías y sindicatos.

La mayoría de los autores apuestan por aplicar mecanismos de gestión empresarial a las organizaciones no lucrativas o lo que es lo mismo, buscar modelos de negocio que hagan sostenible la búsqueda del beneficio social (Reis y otros, 2001). En muchas ocasiones la implementación de estos instrumentos de mercado se torna difícil debido a la propia naturaleza de estas organizaciones, con estructuras muy diferentes a las empresas privadas. Para superar estas dificultades se suele hacer uso de procesos de innovación en la gestión y los modelos de negocio de este tipo de empresas (Nicholls y Cho, 2006).

Según la literatura revisada, las características principales que definen a la empresa social y la diferencian del resto serían las siguientes:

a) La misión o el objetivo principal: sería una seña de identidad de la empresa social, en la medida en que desde el comienzo de la puesta en marcha de la empresa (emprendimiento) el objetivo principal sea social, aunque para ello deba haber un balance económico positivo (Boschee, 1998).

b) La creación de valor compartido: esta teoría surge de la concepción del conocido investigador de la Universidad de Harvard, Michael Porter. Según esta teoría (Porter y Kramer, 2011), las empresas deben aliarse de nuevo con la sociedad, como si hubieran perdido su orientación fundamental y su razón de ser, que está precisamente en ser útiles a las personas. Se supera por tanto el clásico concepto de filantropía corporativa, donde la empresa medía el rédito ecocómico de sus actividades de acción social (Mescon, T., Tilson, D., 1987; Françoise, L., 1995). El concepto de valor compartido se refiere a las políticas y prácticas operacionales que mejoran la competitividad de una empresa a la vez que ayudan a mejorar las condiciones económicas y sociales en las comunidades donde opera. La creación de valor compartido requiere del cumplimiento de estándares éticos pero va más allá porque supone una nueva forma de entender la eficacia y la eficiencia de la empresa centrándose en las necesidades de las personas. La aplicación de esta teoría implica una reconversión del concepto clásico de empresa dirigido a maximizar beneficios y por tanto invita a reestructurar las políticas de responsabilidad social empresarial hacia la centralidad de crear valor compartido.

c) Medición de impactos: medir el impacto quiere decir utilizar métricas para asignar un valor los objetivos que se están cumpliendo. Sin embargo, hay 
todavía grandes deficiencias en las herramientas de medición del impacto social, sobre todo teniendo en cuenta la dificultad que entraña medir intangibles e identificar los los inputs/outputs que genera la actividad de la empresa social (Kramer y otros, 2009). La medición, por otra parte, debería hacerse en la conocida triple línea de lo social, lo económico y lo medioambiental (Fernández, 2012). En este sentido, una de las últimas iniciativas pasa por utilizar la teoría del cambio como instrumento de medición. Esta teoría relaciona el problema social con el conjunto de actividades e impactos que por definición debería influir sobre el problema anteriormente identificado. El uso de este tipo de instrumentos, según algunos garantizarían el éxito del proceso de emprendimiento social (Lariú y Strandberg, 2009; Shari y Lerner, 2006).

Un objetivo clave de las empresas sociales es tener cierto grado de autosostenibilidad e independencia respecto de agentes externos. Para conseguir la autonomía y la autosuficiencia, las empresas sociales deben utilizar principios comunes en el mundo empresarial: eficiencia (Bagnoli y Megali, 2011); vigilancia de la competencia, el desarrollo de ventajas competitivas captar clientes e inversores (Yunus, 2008); gestión del riesgo económico (Defourny, 2001); eficiencia de sus estructuras y eficacia a la hora de conseguir y gestionar sus recursos (Dees, 1998). Las dificultades con las que se encuentran día a día las organizaciones sociales hacen que este escenario no sea así y que, en muchas ocasiones, las empresas sociales solo puedan cubrir sus costes de manera parcial, compitiendo en desventaja con otro tipo de empresas tradicionales (Dees, 2008).

Esta situación de dificultad puede ocurrir como consecuencia de múltiples causas. A veces, la misión social que persiguen este tipo de empresas conlleva una baja productividad de bienes y servicios. Por ejemplo, pudiera darse el caso de una organización, en base a unos principios determinados, se constituyera con un régimen de propiedad compartida o solidaria, donde la toma de decisiones se puede tornar más lenta. $\mathrm{O}$ las empresas que provean acceso a bienes básicos a colectivos de bajos ingresos verán mermados sus márgenes al tener que ofrecer unos precios muy bajos para satisfacer a sus clientes (Prahalad, 2002).

Según Bagnoli y Megali (2011), las empresas sociales son ante todo, empresas y deben perseguir sus objetivos sociales respetando siempre la eficiencia económica y financiera. Si una empresa social opera temporalmente en pérdidas, debiera tener una justificación social para ello. Por último, la poca visibilidad de estas organizaciones hace que no se haya consolidado un mercado de capitales en el que puedan obtener recursos para financiar su actividad. En este último punto, cabe destacar la aportación de Yunus (2011) fundador del Grammeen Bank, que precisamente aportaba microcréditos a emprendedores sociales debido a la falta de un mercado de capital que invirtiera en este tipo de proyectos orientados a la generación de valor social. 
Las empresas sociales no dependen exclusivamente de sus ventas, sino que combinan éstas con otras fuentes no comerciales, como donaciones, subvenciones, etcétera. Esta dependencia en los ingresos propios puede variar en gran medida desde una dependencia pequeña hasta la deseada dependencia total (Campbell, 1998).

A la hora de entender a sus grupos de interés, sobre todo a la comunidad donde opera o que es beneficiaria, la empresa social tiene muy en cuenta las exigencias y la propia cultura de la comunidad, la realidad que supone y los impactos que puede generar su modelo de negocio (Campbell, 1998). En ocasiones se lleva al extremo, y el emprendedor social busca implicarse con los propios problemas de la comunidad, extendiendo su iniciativa a la participación de todos los beneficiarios. Para ello usa, como veremos a continuación en el apartado siguiente, la innovación social, destinada a contribuir a cambios por y para la comunidad (Mair y Marti, 2006). Algunos autores han apuntado que más allá de un nuevo modelo empresarial, este tipo de empresas actúa de palanca de cambio para que las empresas tradicionales tengan más en cuenta los impactos y los efectos que su actividad ejerce en los diferentes grupos de interés (Bajo, 2011).

Para afrontar el estudio de la empresa social, el investigador habrá de prestar atención a las teorías de organización, las teorías de gestión, management y todas las subteorías o disciplinas del ámbito de la ciencia empresarial. Hoy día los aspectos que más interés despiertan a tenor del número de publicaciones, son los que tienen que ver con la medición de los intangibles, la generación del valor y las alianzas que este tipo de organización establecen (Short y otros, 2009).

\subsection{Escuela de la innovación social}

Hay varias maneras de entender la innovación como tal. La más clásica es la de Shumpeter (1996) que la definió como la introducción de nuevos métodos de producción o la apertura a nuevos mercados. Otros la entienden como el intento de llevar a la práctica una idea novedosa con éxito, lo que exige gran esfuerzo y compromiso por parte de la organización (Alvord y otros, 2004). Por otro lado, algunos investigadores ofrecen una visión más amplia del término y lo extienden también al conjunto de organizaciones innovadoras, cuando operan cambios o modificaciones en cualesquiera de sus procesos o actividades (Godin, 2006). Dichos cambios requieren con frecuencia nuevos modos de gestión.

Desde la década de los 70, el concepto de innovación social se ha relacionado con instituciones públicas y privadas, universidades y organizaciones del tercer sector. Con todo, el concepto aún adolece de precisión. De todas formas merece destacar como en cierta literatura se le suele encontrar relacionado con el concepto de emprendimiento social, toda vez que este último requiere de una alta capacidad de innovación. 
Según la definición del Oxford Said Business School, "la innovación social se refiere a nuevas ideas que funcionan en el cumplimiento de beneficios sociales" (Mulgan, y otros 2007: 8). Las organizaciones que buscan objetivos sociales con instrumentos del mercado, necesitan de procesos y métodos innovadores para llevar a cabo con éxito su misión. Para ello cuentan, por ejemplo, con la inteligencia colectiva, a través de mecanismos de participación abierta. Las redes de contacto y colaboraciones también son fundamentales para reducir costes y compartir conocimiento. La interacción con los grupos de interés debe ser cada vez más cercana y así el producto final o la manera de resolver las necesidades será más sencilla para la organización. En los últimos años, la innovación social ha crecido de manera considerable y la encontramos sobre todo enfocada a circunstancias muy determinadas de nuestro mundo: el aumento de la esperanza de vida para las personas más vulnerables; el aumento de la diversidad en muchos países y ciudades debido a los movimientos migratorios; las desigualdades que produce esta situación y las diferencias de renta entre los países del norte y los del sur; los riesgos de las enfermedades de larga duración derivadas de las malas condiciones de vida y alimentación; las transiciones difíciles a la edad adulta, como adicciones o problemas de salud mental; o las situaciones de conflicto político y bélico (Mulgan, y otros 2007)

Para Irizar (2008) el término de innovación social se refiere al proceso a través del cual las diferentes necesidades de cambio o ideas se convierten en realidades políticas o de servicio público. Para otros investigadores, por el contrario, la línea de separación entre lo público y lo privado no es tan evidente cuando nos referimos a los cambios sociales (Johnson, 2000). Hay quienes lo relacionan con los procesos de innovación tecnológicos y las herramientas de las tecnologías de la información y la comunicación (TICs). Suele hacerse hincapié en que la puesta en marcha de elementos de esta índole podría resultar útil en la lucha contra la exclusión social y para generar desarrollo económico y progreso social (Morales, 2008).

Uno de los artículos más citados al respecto es el de Waddock y Post (1991), donde analizan la innovación social desde el punto de vista del agente de cambio social y político. Para estos autores, el emprendimiento social, y la innovación como proceso, es un catalizador para el cambio. Estos emprendedores analizarían los problemas y les darían solución desde lo público, identificando los mecanismos para su correcto uso e implementando procesos de mejora constante.

A diferencia de la anterior perspectiva, algunos autores afirman que la innovación social lleva implícita la adaptación de las ideas de cambio social al mercado, a través de redes de confianza; y la colaboración o la creación de mecanismos de participación ciudadana (Murray y otros, 2011). En este sentido, las innovaciones sociales se cuentan como iniciativas a favor de colectivos vulnerables $\sin$ capacidad de recrear ecosistemas propicios para el desarrollo comunitario (Pome- 
rantz, 2003). Un buen ejemplo sería la incipiente economía colaborativa o una alternativa a la banca tradicional, la banca ética (Ballesteros, 2005). No tiene por qué llevar implícito un modelo de negocio que busque maximizar el valor comercial, y no siempre se trata de organizaciones con dirección centralizada, sino que más bien hablaríamos de conectores o instrumentos al servicio del cambio social. La interacción ciudadana y comunitaria debe producir sinergias positivas e implicar procesos de innovación también en las organizaciones, empresas e incluso en las entidades públicas, de ahí la importancia del trabajo en red (Abreu, 2011).

Esta aproximación al emprendimiento social que subraya la necesidad de llevar a cabo procesos creativos y de innovación transformadora del sistema en su conjunto constituye, sin duda, el enfoque más retador y que mayor espacio abre a la reflexión y a la investigación. Cabe esperar que desde esta sensibilidad se vayan pudiendo anticipar tendencias y elaborar propuestas razonables desde las que enfrentarse a las múltiples caras de la agenda global: cambio climático, desigualdad, pobreza, etc.

\section{CONCLUSIONES}

La primera misión de todo investigador que se enfrenta de forma científica al estudio de un tema es conocer la naturaleza del objeto de estudio, es decir el problema ontológico. En el ámbito de las ciencias sociales y humanas, los límites de la ciencia no siempre aparecen de manera evidente, sino que el investigador debe hacer uso de las herramientas epistemológicas para saber cómo enfrentarse a ellos desde una perspectiva académica. El principal problema que encontramos cuando nos acercamos al fenómeno del emprendimiento social es la falta de consenso a la hora de definirlo. Dependiendo del autor el enfoque es diferente. No existe por tanto una definición definitiva a nivel académico. Este hecho justifica la necesidad de hacer un marco teórico que describa las diferentes definiciones, mediante una revisión de la literatura más relevante.

El objetivo principal de este trabajo ha sido, precisamente, el plantear los supuestos taxonómicos de manera que se consiga una división clara entre las fronteras teóricas de un fenómeno complejo como el del emprendimiento social. La revisión de la literatura más relevante ha permitido clasificar los conceptos basándonos en una triple dimensión epistemológica, a modo de escuelas de pensamiento, a saber: (i) la escuela del emprendedor social: comprende la dimensión micro, es decir el individuo que emprende, el estudio de sus motivaciones, aspiraciones, valores y tendencias sociológicas; (ii) la escuela de la empresa social: el estudio desde el enfoque de la organización, la dimensión meso y comprende el estudio de los modelos organizativos o teorías de management; por último, (iii) la escuela de la innovación social, que hace referencia a la interrelación de las dos 
anteriores con el sistema político, económico, social y medioambiental, donde hablamos de la dimensión macro.

En la escuela del emprendedor social, los autores coinciden en la perspectiva de actor de cambio del sujeto que emprende. Las condiciones para este reconocimiento descansan en la capacidad de innovación y la manera en que se enfrenta al problema que quiere resolver. Más allá de las competencias o habilidades, se debe demostrar una vocación de servicio y la creación de valor social. La relación de este emprendedor con la sostenibilidad se ha puesto de manifiesto, en tanto en cuanto el emprendedor social busca solucionar problemas de manera sostenible, escalando la creación de valor social para que beneficie al mayor número de personas.

Las mayores divergencias las encontramos en la escuela de la empresa social. Para algunos tiene que ver con el mundo del tercer sector o la economía social, es decir, hablamos de organizaciones sin ánimo de lucro. Para otros, el enfoque dominante es un híbrido entre la búsqueda del objetivo social con el uso de herramientas e instrumentos del mercado, entrando en la esfera del mercado de competencia. La medición del impacto y la orientación a la creación de valor compartido, son algunas de las líneas de investigación que más interés están suscitando entre la comunidad científica.

Por último, la escuela de la innovación social cierra el triángulo de las tres escuelas en que hemos clasificado el fenómeno para su comprensión y clarificación. Para los principales autores, el término de innovación social tiene que ver con la manera en que las ideas se ponen al servicio del cambio social. Para los procesos de innovación social tan importante es la manera en que se enfocan los problemas como los resultados que esos cambios suponen para la mejora de la calidad de vida de las personas, sobre todo las más vulnerables y apartadas del sistema económico imperante. De ahí que muchos autores lo relacionen con los cambios generados en las políticas públicas y las instituciones gubernamentales.

Las principales conclusiones tienen que ver con la manera de afrontar el estudio de un fenómeno tan complejo como este y que tanto interés está despertando en diferentes escuelas académicas. La mayoría de los enfoques viene desde el mundo de la ciencia económica y empresarial, y tiene que ver con la necesidad y la responsabilidad social de la academia por aportar alternativas a modelos productivos y sistemas socioeconómicos que han entrado en crisis y generan desigualdad y pobreza. Pero a su vez la mayor parte de autores entienden el emprendimiento social como una oportunidad para reparar el sistema capitalista abriendo nuevos campos de estudio en el ámbito de las organizaciones que van más allá de gestión con un enfoque de responsabilidad social, sino que desde el momento de su fundación (emprendimiento) la cultura de la organización se fundamenta bajo valores éticos que se aplican de forma proactiva, como la justicia o la igualdad. 
Debemos seguir profundizando, tanto en la descripción como en la explicación de las tres escuelas desarrolladas aquí. Las líneas de estudio más interesantes se pueden conocer a través de dos vías fundamentalmente. Por un lado el interés que la comunidad académica tiene sobre un tema en concreto a tenor de los estudios bibliométricos consultados y por otro lado por las lagunas que hemos detectado a la hora de hacer la revisión bibliográfica. La primera de estas líneas pone el foco en los fundamentos éticos y filosóficos del fenómeno del emprendimiento social. Se ve necesario aplicar las teóricas de la ética empresarial y la ética aplicada para discernir sobre cómo puede estar enfrentándose el emprendimiento social a los dilemas y problemáticas humanos de hoy en día a diferentes niveles. También es necesario el análisis de la realidad, es decir, el estudio empírico (cualitativo o cuantitativo) para saber cómo las empresas o los emprendedores sociales (dependiendo la escuela) están resolviendo los retos o problemas sociales: por decirlo de otra manera, cuáles son las claves del éxito de las empresas y los emprendedores sociales, las barreras que están impidiendo el desarrollo de estas empresas. Por último, detectamos una interesante línea de investigación en el tema de la financiación de las empresas sociales. No hemos encontrado muchos estudios acerca de la inversión de impacto y de cómo las empresas sociales se están adaptando a las exigencias de estos inversores con cierta conciencia social, que no solo buscan la rentabilidad económica de sus inversiones.

\section{BIBLIOGRAFÍA}

ABREU, J. L. (2011), "Innovación social: conceptos y etapas", Daena: International Journal of Good Conscience, vol. 6, núm. 2, pp. 134-148.

ALVORD, S. H. y otros (2004), "Social entrepreneurship and societal transformation an exploratory study", The journal of applied behavioral science, vol. 40, núm. 3, pp. 260-282.

AUSTIN, J., y otros (2006), "Social and comercial entrepreneurship: Same, different, or both?", Entrepreneurship: Theory and Practice, vol. 30, núm. 1, pp. 1-22.

BAGNOLI, L. y MEGALI, C. (2011), "Performance measuring in social enterprises", Nonprofit Volunt. Sect. Q, vol. 40, núm. 1, pp. 149-165.

BAJO, A. (2011), "Pasos hacia un cambio de Paradigma", en A. Bajo y N. Villagra (eds.) Los grandes retos de la empresa en el siglo XXI, Universidad Pontificia Comillas, Madrid, pp. 179-190.

BAJO, A. (2012), "La insostenibilidad en torno a la sostenibilidad", Foreign Policy, núm. 59, Disponible en: http://energuia.com/wp-content/uploads/ kalins-pdf/singles/la-insostenibilidad-en-torno-a-la-sostenibilidad.pdf (acceso: 01/12/2014). 
BALLESTEROS, C. (2005), "La banca ética", en A. Federico, R. Muñoz y S. Ozomek (comps.). Finanzas y economía social. Modalidades en el manejo de los recursos solidarios. OSDE-UNGS, Editorial Altamira, Buenos Aires, pp. 307-408.

BENAVIDES, J. y otros (2012), Glosario sobre Responsabilidad Social para la investigación y el debate terminológico, Universidad Pontificia Comillas, Madrid.

BLOOM, P. y CHATTERJI, A. (2009), "Scaling social entrepreneurial impact", California management review, vol. 51, núm. 3, pp. 114-133.

BORNSTEIN, D. (2004), How to change the world: Social entrepreneurs and the power of new ideas, Oxford University Press, Oxford.

BOSCHEE, J. (1998), Merging mission and money: A board member's guide to social entrepreneurship, National Center for Nonprofit Boards, Washington.

CAMPBELL, S. (1998), "Social entrepreneurship: how to develop new social-purpose business ventures", Health care strategic management, vol. 15, núm. 5, p. 17.

CASTRO, L. y TORO, D. (1996), Metodología de las ciencias sociales. Una introducción crítica, Tecnos, Madrid.

CAVAGNARO, E. y CURIEL, G. (2012), The three levels of Sustainability, Greenleaf Publishing Limited, Shefield.

CHÁVEZ, R. (2012), "Social Economy, an international perspective: 25 years of CIRIEC-España”, Revista de economía pública, social y cooperativa, núm. 73, pp. 5-8.

CHÁVEZ, R. y MONZÓN, J. L. (2001), "Trajectoires des groupes d'économie sociale", en D. Coté (Dir.), Les holdings cooperatifs: évolution ou transformation definitive?, De Boeck-Waesmael - Ciriec, Bruxelles, pp. 53-76.

COMISIÓN EUROPEA, (2011), La Iniciativa de Emprendimiento Social de la Comisión Europea, http://ec.europa.eu/internal_market/publications/docs/ sbi-brochure/sbi-brochure-web_es.pdf (acceso 05/01/2015).

DACIN, P. A. y otros (2010), "Social entrepreneurship: Why we don`t need a new theory and how we move forward from here", Academy of Management Perspectives, vol. 24, núm. 3, pp. 37-57.

DEES, G. y ANDERSON, B. (2004), "Scaling social impact: strategies for spreading social innovation", Stanford Social Innovation Review, vol. 1, núm. 4, pp. 24-32.

DEES, G. (1998), "The meaning of "Social Entrepreneurship", Fuqua School of Business. Duke's University, pp. 1-6. Disponible en: http://www.redalmarza.cl/ing/pdf/TheMeaningofsocialEntrepreneurship.pdf (acceso: 25/09/2013).

DEES, G. (2008), "Philanthropy and enterprise: Harnessing the power of business and social entrepreneurship for development", Innovations, vol. 3, núm. 3, pp. 119-132. 
DEFORUNY, J. y NYSSENS, M. (2010), "Conceptions of social enterprise and social entrepreneurship in Europe and the United States: convergences and divergences", Journal of social entrepreneurship, vol. 1, núm. 1, pp. 32-53.

ESPÍRITU, R. y SASTRE, C. (2007), "La actitud emprendedora durante la vida académica de los estudiantes universitarios", Cuadernos de estudios empresariales, núm. 17, pp. 95-116.

FELBERG, C. (2012), La economía del bien común, Editorial Deusto, Bilbao.

FERNÁNDEZ, J. L. (2012), Investigar para un mundo sostenible. Lección inaugural del Curso Académico 2012-2013, Universidad Pontificia Comillas, Madrid.

FRANÇOISE, L. (1995), "Global corporate philanthropy: a strategic framework", International Marketing Review, vol. 12, núm. 4, pp. 20-37.

GIDDENS, A. (1998), La constitución de la sociedad: bases para la teoría de la estructuración, Amorrortu editores, Madrid.

GODIN, B. (2006), "The Linear model of innovation the historical construction of an analytical framework", Science, Technology $\mathcal{E}$ Human Values, vol. 31, núm. 6, pp. 639-667.

HIBBERT, S. A. y otros. (2002), Consumer response to social entrepreneurship: The case of the Big Issue in Scotland. International Journal of Nonprofit and Voluntary Sector Marketing, vol. 7, núm. 3, pp. 288-301.

JOHNSON, S. (2000), "Literature review on social entrepreneurship", Canadian Centre for Social Entrepreneurship, vol. 16, p. 16.

KRAMER, M., y otros (2009), Breakthroughs in shared measurement and social impact, FSG Social Impact Advisors, Boston.

LARIÚ, A. y STRANDBERG, L. (2009), "Negocios inclusivos: Creando valor para las empresas y para la población de bajos ingresos", Cátedra "la Caixa" de Responsabilidad Social de la Empresa y Gobierno Corporativo. Disponible en: http://www.iese.edu/en/files/cuaderno\%20no\%205_tcm439620.pdf (acceso: 7/09/2013).

LIGHT, P. C. (2006), "Reshaping social entrepreneurship", Stanford Social Innovation Review, vol. 4, núm. 3, pp. 47-51.

MADDEN, T. J. y otros (1992), "A comparison of the theory of planned behavior and the theory of reasoned action", Personality and social psychology Bulletin, vol. 18, núm. 1, pp. 3-9.

MARTIN, R. J. y OSBERG, S. (2007), "Social entrepreneurship: The case for a definition", Stanford Social Innovation Review, vol. 5, núm. 2, pp.29-39.

MAIR, J. y MARTI, I. (2006), "Social entrepreneurship research: A source of explanation, prediction, and delight", Journal of World Business, vol. 41, núm. 1, p. 36.

MARTÍNEZ, J. (2005), "La dignidad humana en la doctrina social de la Iglesia”, en J. Martínez, C, Perrotin, y F. Torralba, Repensar la dignidad humana, Editorial Milenio, Lleida, pp. 93-162. 
MESCON, T., TILSON, D. (1987), "Corporate Philanthropy: A strategic approach to the Bottom-Line", California Management Review, vol. 29, núm. 2, pp. 49-61.

MERINO, A. (2014), "El emprendimiento social: un territorio de aprendizaje para la superación de un modelo en crisis", Revista ICADE, núm. 91, pp. 181-182.

MULGAN, G. y otros (2007), Social innovation: what it is, why it matters and how it can be accelerated, The Basingstoke Press, London.

MURRAY, R. y otros (2011), The open book of social innovation. National Endowment for Science, Technology and the Art. Disponible en: http://desisdop.org/documents/10157/12818/Murray,+CaulierGrice,+Mulgan+(2010), +The+Book+of+Social+Innovation.pdf (acceso: 3/10/2014)

NECK, H., y otros (2009), "The landscape of social entrepreneurship", Business Horizons, vol. 52, núm. 1, pp. 13-19.

NICHOLLS, A., CHO, A. H. (2006), "Social entrepreneurship: The structuration of a field. Social entrepreneurship", New models of sustainable social change, núm. 1, pp. 99-118.

PAREJA, B. (2013), "El emprendimiento social: una introducción a los conceptos, dimensiones y teorías", Revista de servicios sociales y política social, núm. 102, pp.11-22.

PAREJA, B. (2013a), "Perspectiva multidisciplinar del emprendimiento social", en A. Bajo y N. Villagra (eds.), La responsabilidad social en el ámbito de la crisis: memoria académica curso 2012-2013, Universidad Pontificia Comillas, Madrid, pp.173-204.

PEREDO, A. M. y MCLEAN, M. (2005), "Social entrepreneurship: A critical review of the concept", Journal of world business, 41(1), pp. 56-65.

PÉREZ DE MENDIGUREN, J. C., y otros (2008), “De qué hablamos cuando hablamos de Economía Social y Solidaria? Concepto y nociones afines", Comunicación presentada a las XI Jornadas de Economía Crítica, Bilbao, 27-29 de marzo

PORTER, M. E. and KRAMER, M. R. (2011), "Creating shared value", Harvard Business Review, vol. 89, núm. 1, pp.1-17.

PRAHALAD, C. K. (2002), "Serving the world's poor, profitably", Harvard business review, vol. 80, núm. 9, pp. 48-59.

POMERANTZ, M. (2003), "The business of social entrepreneurship in a "down economy", Business, vol. 25, núm. 3, pp. 25-30.

PRASZKIER, R. and NOWAK, A. (2012), Social Entrepreneurship. Theory and practice, Cambridge University Press, Cambridge.

REIS, T. K., y otros (2001), "Unleashing new resources and entrepreneurship for the common good: A philanthropic renaissance", New Directions for Philanthropic Fundraising, vol. 32, pp. 109-144.

SCHUMPETER, J. (1996), Historia del análisis económico, Ariel, Madrid. 
SHANE, S. A. and VENKATARAMAN, S. (2000), "The promise of entrepreneurship as a field of research", Academy of Management Review, vol. 25, pp. 217-226.

SHAPERO, A. and SOKOL, L. (1982), "The social dimensions of entrepreneurship", Encyclopedia of entrepreneurship, University of Arizona, Tucson, pp. 72-90.

SHARI, M. and LERNER, G. (2006), "Gauging the success of social ventures initiated by individual social entrepreneurs", Journal of World Business, núm. 41, pp. 6-20.

SHORT, J. C., y otros (2009), "Research in social entrepreneurship: Past contributions and future opportunities", Strategic entrepreneurship journal, vol. 3, núm. 2, pp. 161-194.

SMITH, B. and STEVENS, C. (2010), "Different types of social entrepreneurship: The role of geography and embeddedness on the measurement and scaling of social value", Entrepreneurship and Regional Development, vol. 22, núm. 6, pp. 575-598.

SULLIVAN, G. y otros (2003), "Social entrepreneurship: Towards conceptualization", International journal of nonprofit and voluntary sector marketing, vol. 8, núm. 1, pp. 76-88.

THOMPSON, J. y otros (2000), "Social entrepreneurship: a new look at the people and the potential", Management decision, vol. 38, núm. 5, pp. 328-338.

URTEAGA, E. y EIZAGIRRE, A. (2011), El nuevo entorno de la innovación: sostenibilidad y legitimación social, Ediciones de la Universidad de Oviedo, Oviedo.

WADDOCK, S. A. y POST, J. E. (1991), "Social entrepreneurs and catalytic change", Public administration review, núm. 51, pp. 393-401.

YUNUS, M. (2008). Un mundo sin pobreza, Editorial Paidós, Barcelona.

YUNUS, M. (2011), Las empresas sociales. Una nueva dimension del capitalism para atender las necesidades más acuciantes de la humanidad, Editorial Paidós, Barcelona.

ZAHRA, S. A. y otros (2009), "A typology of social entrepreneurs: Motives, search processes and ethical challenges", Journal of business venturing, 24(5), pp. 519-532.

ZHAO, H. y otros (2005), "The mediating role of self-efficacy in the development of entrepreneurial intentions", Journal of Applied Psychology, núm. 90, pp. 1265-1272.

ZAMAGNI, S. (2012), Por una economía del bien común, Ciudad Nueva, Madrid. 


\section{ANEXO}

Tabla 1. Clasificación de las tres escuelas del emprendimiento social

\begin{tabular}{|c|c|c|}
\hline \multicolumn{3}{|c|}{ ESCUELA DEL EMPRENDEDOR SOCIAL } \\
\hline Autor/es & Definiciones & Año \\
\hline $\begin{array}{c}\text { WADDOCK, S. A. } \\
\text { y POST, J. E. }\end{array}$ & $\begin{array}{c}\text { La creación o la elaboración de una organización } \\
\text { pública con el fin de alterar en gran medida el patrón } \\
\text { existente de la asignación de los escasos recursos } \\
\text { públicos }\end{array}$ & 1991 \\
\hline DEES, G. & $\begin{array}{c}\text { El emprendedor social es un agente de cambio con una } \\
\text { misión social y una visión innovadora }\end{array}$ & 1998 \\
\hline WALLACE, S. L & $\begin{array}{c}\text { Emprendedores con una responsabilidad social por } \\
\text { mejorar aquellas comunidades donde opera. }\end{array}$ & 1999 \\
\hline LIGHT, P. C. & $\begin{array}{c}\text { Un emprendedor social es un individuo, grupo, } \\
\text { organización o alianza de organizaciones que de forma } \\
\text { sostenible busca un cambio a gran escala introduciendo } \\
\text { nuevas ideas en gobiernos, organizaciones sin ánimo de } \\
\text { lucro, empresas para conducir problemas sociales graves }\end{array}$ & 2006 \\
\hline $\begin{array}{c}\text { MARTIN, R. L. } \\
\text { y OSBERG, S. }\end{array}$ & $\begin{array}{c}\text { El emprendedor social es la persona que se inspira en } \\
\text { las necesidades de marginación o sufrimiento social } \\
\text { para enfocar su acción en buscar un nuevo equilibrio } \\
\text { para el bien de la comunidad o grupo social afectado, } \\
\text { usando para ello la creatividad. }\end{array}$ & 2007 \\
\hline
\end{tabular}

\begin{tabular}{|c|c|c|}
\hline \multicolumn{3}{|c|}{ ESCUELA DE LA EMPRESA SOCIAL } \\
\hline Autor/es & Definiciones & Año \\
\hline CAMPBELL, S. & $\begin{array}{c}\text { Proporcionar a las comunidades productos o servicios } \\
\text { que necesitan y generan ganancias para apoyar las } \\
\text { actividades que no pueden generar ingresos. }\end{array}$ & 1998 \\
\hline $\begin{array}{c}\text { HIBBERT, S. A., } \\
\text { y otros. }\end{array}$ & $\begin{array}{c}\text { El uso de comportamiento emprendedor tanto para } \\
\text { fines sociales como para objetivos de negocio, o una } \\
\text { empresa que genera beneficios atendiendo a un grupo } \\
\text { social excluido }\end{array}$ & 2002 \\
\hline AUSTIN, y otros & $\begin{array}{c}\text { El emprendimiento social es una actividad innovadora, } \\
\text { creadora de valor social que puede llevarse a cabo a } \\
\text { través del sector no lucrativo de negocios o a nivel de } \\
\text { gobiernos y entidades públicas }\end{array}$ & 2006 \\
\hline
\end{tabular}




\begin{tabular}{|c|c|c|}
\hline \multicolumn{3}{|c|}{ ESCUELA DE LA EMPRESA SOCIAL } \\
\hline Autor/es & Definiciones & Año \\
\hline $\begin{array}{c}\text { NICHOLLS, A. } \\
\text { y CHO, A. H }\end{array}$ & $\begin{array}{l}\text { Implica innovaciones destinadas a mejorar de forma } \\
\text { explícita bienestar social, alojados dentro de las } \\
\text { organizaciones empresariales que inician, guía o } \\
\text { contribuir al cambio en la sociedad. }\end{array}$ & 2006 \\
\hline $\begin{array}{l}\text { MAIR, J. } \\
\text { y MARTI, I. }\end{array}$ & $\begin{array}{c}\text { Emprendimiento social: modelos innovadores } \\
\text { de proveer productos y servicios que abastezcan } \\
\text { las necesidades básicas (derechos) que quedan } \\
\text { insatisfechos por las instituciones políticas o } \\
\text { económicas. }\end{array}$ & 2006 \\
\hline $\begin{array}{l}\text { NECK, H. } \\
\text { y otros. }\end{array}$ & $\begin{array}{l}\text { La diferencia de un emprendimiento social de otro } \\
\text { tradicional es la preeminencia de la misión social del } \\
\text { primero. }\end{array}$ & 2009 \\
\hline $\begin{array}{l}\text { DEFOURNY, J. } \\
\text { y NYSSENS, M. }\end{array}$ & $\begin{array}{l}\text { Nuevos comportamientos empresariales impulsados } \\
\text { por un propósito social primario y que se llevaron } \\
\text { a cabo en un primer momento en el tercer sector, } \\
\text { abarcando más adelante a otro tipo de organizaciones } \\
\text { empresariales y sociales. }\end{array}$ & 2010 \\
\hline
\end{tabular}

\begin{tabular}{|c|c|c|}
\hline \multicolumn{3}{|c|}{ ESCUELA DE LA INNOVACIÓN SOCIAL } \\
\hline Autor/es & Definiciones & Año \\
\hline JOHNSON, S & $\begin{array}{l}\text { El emprendimiento social está surgiendo como un } \\
\text { enfoque innovador para hacer frente a las necesidades } \\
\text { sociales complejas. Con su énfasis en la resolución } \\
\text { de problemas y la innovación social, las actividades } \\
\text { empresariales socialmente desdibujan las fronteras } \\
\text { tradicionales entre el sector público, privado y sector sin } \\
\text { fines de lucro, y hacen hincapié en el modelo híbrido de } \\
\text { actividades con fines de lucro y sin fines de lucro. }\end{array}$ & 2000 \\
\hline $\begin{array}{l}\text { SULLIVANWa, G., } \\
\text { y otros. }\end{array}$ & $\begin{array}{l}\text { La búsqueda y el reconocimiento de las oportunidades } \\
\text { que conducen a la creación de nuevas organizaciones } \\
\text { sociales y la continua innovación en los ya existentes. }\end{array}$ & 2003 \\
\hline POMERANTZ, M. & $\begin{array}{l}\text { Tratar de aprovechar la innovación para enfocarlas a } \\
\text { cubrir necesidades o problemas sociales. }\end{array}$ & 2003 \\
\hline $\begin{array}{l}\text { PEREDO, A. M. y } \\
\text { McLEAN, M. }\end{array}$ & $\begin{array}{c}\text { Es un instrumento para diseñar respuestas ante las } \\
\text { necesidades sociales, por lo tanto es útil para diseñar } \\
\text { políticas sociales. }\end{array}$ & 2005 \\
\hline
\end{tabular}

Fuente: elaboración propia a partir de la literatura. 